\title{
Graphene Oxide-Based Layered Bulk Material Reinforced by Amorphous/Crystalline Heterophase Platelets and Multiscale Interface Crosslinking
}

\section{Ke Chen}

Key Laboratory of Bio-Inspired Smart Interfacial Science and Technology of Ministry of Education, School of Chemistry, Beihang University (BUAA)

\section{Xuee Tang}

Key Laboratory of Bio-Inspired Smart Interfacial Science and Technology of Ministry of Education, School of Chemistry, Beihang University (BUAA)

\section{Binbin Jia}

Key Laboratory of Bio-Inspired Smart Interfacial Science and Technology of Ministry of Education, School of Chemistry, Beihang University (BUAA)

\section{Cezhou Chao}

School of Aeronautic Science and Engineering, Beihang University

\section{Junyu Hou}

Beihang University

\section{Leiting Dong}

School of Aeronautic Science and Engineering, Beihang University

\section{Yan Wei}

Peking University

\section{Ting-Hui Xiao}

University of Tokyo https://orcid.org/0000-0002-7339-152X

\section{Keisuke Goda}

Department of Chemistry, The University of Tokyo

\section{Lin Guo ( $\square$ guolin@buaa.edu.cn )}

Beihang University https://orcid.org/0000-0002-6070-2384

\section{Article}

Keywords: Graphene oxide, reduced-GO, layered bulk material, structural materials

Posted Date: August 24th, 2021

DOI: https://doi.org/10.21203/rs.3.rs-807031/v1 
License: (c) (i) This work is licensed under a Creative Commons Attribution 4.0 International License. Read Full License

Version of Record: A version of this preprint was published at Nature Materials on July 7th, 2022. See the published version at https://doi.org/10.1038/s41563-022-01292-4. 


\section{Graphene Oxide-Based Layered Bulk Material Reinforced by Amorphous/Crystalline Heterophase Platelets and Multiscale Interface Crosslinking}

Ke Chen ${ }^{1}$, Xuke Tang ${ }^{1}$, Binbin $\mathrm{Jia}^{1}$, Cezhou Chao ${ }^{2}$, Junyu Hou ${ }^{1}$, Leiting Dong ${ }^{2, *}$, Yan $\mathrm{Wei}^{3, *}$, Ting-Hui Xiao ${ }^{4}$, Keisuke Goda ${ }^{4,5,6}$, and Lin Guo ${ }^{1, *}$

1. Beijing Advanced Innovation Center for Biomedical Engineering, Key Laboratory of Bio-Inspired Smart Interfacial Science and Technology of Ministry of Education, School of Chemistry, Beihang University (BUAA), Beijing 100191, China.

2. School of Aeronautic Science and Engineering, Beihang University (BUAA), Beijing 100191, China.

3. Department of Geriatric Dentistry, NMPA Key Laboratory for Dental Materials, National Engineering Laboratory for Digital and Material Technology of Stomatology, Beijing Laboratory of Biomedical Materials, Peking University School and Hospital of Stomatology, Peking University, Beijing 100081, China.

4. Department of Chemistry, The University of Tokyo, Tokyo 113-0033, Japan.

5. Institute of Technological Sciences, Wuhan University, Hubei 430072, China.

6. Department of Bioengineering, University of California, Los Angeles, California 90095, United States.

These authors contributed equally: Ke Chen, Xuke Tang, Binbin Jia, Cezhou Chao Email: guolin@buaa.edu.cn, ltdong@buaa.edu.cn, kqweiyan@bjmu.edu.cn

Graphene oxide (GO) or reduced-GO offer excellent mechanical, electrical and chemical properties. Their nanocomposites have been increasingly explored for attractive applications in diverse fields. However, due to the flexible feature and weak interlayer interactions of GO sheets, flexural mechanical properties of GObased composites especially for the bulk materials are largely restrained, which would hinder their use in real situations. Here inspired by amorphous/crystalline heterophase features within nacreous platelets, we construct a centimetre-sized GO-based bulk, the building blocks of which consist of crystalline GO and amorphous/crystalline $\mathrm{MnO}_{2}$ phases adhered by polymer-based crosslinkers. The $\mathrm{GO} / \mathrm{MnO}_{2}$ heterophase layers are stacked and hot-pressed with further crosslinking between the layers to form bulk artificial nacre. The resultant $\mathrm{GO} / \mathrm{MnO}_{2}$-based layered (GML) bulk exhibits the highest flexural strength (up to 203.4 MPa) among all of GO-based bulk materials. Moreover, an excellent fracture toughness, a strong impact resistance and light weight are also achieved. Mechanical and simulation analyses corroborate that the highly ordered heterophase structure together with complex crosslinking interactions across multiscale interfaces, lead to superior mechanical properties. We expect that these results provide interesting insights into the design of structural materials and allow the use of high-performance GO-based bulks in engineering and military applications. 
Graphene oxide (GO) nanosheet is well known for its strong mechanical property, excellent biocompatibility, high aqueous dispersibility, rich functionality, and easy oxidative exfoliation from graphite ${ }^{1-5}$. Such advantages make GO nanosheet as a great promising nanofiller to create mechanically superior macroscopic structures. GO and reduced GO-based three-dimensional (3D) bulk assemblies as mechanical and functional parts have potentially widespread applications in aerospace, biomedicine, electronics, military protection, etc ${ }^{2,4}$. However, the direct preparation of self-standing GO-based structures from dispersions or gels has largely been limited to highperformance long fibres ${ }^{5,6}$, thin films (or papers) ) $^{1,3,7-9}$, and lightweight foams (or aerogels $)^{10,11}$. Despite a decade of research efforts, the preparation of GO-based bulk materials with excellent mechanical performance, especially improved flexural mechanical properties, still faces major challenges ${ }^{12,13}$. One of the main barriers lies in a creating strong and controllable micro- and nano-interfaces endows appropriate reinforcement of GO nanosheets or their bridging for jointly strengthening, toughening, and stiffening the assembled bulk nanocomposites, owing to their superior flexibility ${ }^{13,14}$.

The natural structures always inspire the design of materials with attractive mechanical and functional properties. In a typical biomineralization process, living organisms astonishingly control the complex interfaces of their various organo-mineral components to create hierarchical, heterogeneous structural arrangements with intriguing mechanical performance ${ }^{15,16}$. One of the best-known examples is natural nacre, a product of molluscs that has a highly regular 3D 'brick-and mortar' (BM) assembly of ordered aragonite platelets connected by a thin organic interlayer ${ }^{17,18}$. The design, aspect ratio, and interface strength of aragonite platelets allow the nacre strength to be maximized ${ }^{19,20}$. Inspired by nacre structures, many researchers have successfully prepared various types of nacre-like 3D bulks such as impact-resistant glass ${ }^{20}$, ductile ceramics $^{21}$, and superior synthetic nacre ${ }^{22}$. However, there are still critical structural features that have not been considered or duplicated for constructing nacre-mimetic 3D bulks. For example, it is reported that the creation of amorphous/crystalline heterophase within hard mineral tissues, i.e. the platelets, is a pivotal factor for nacreous mechanical contribution, providing an epitaxial match and better adhesion between organic matrix and crystal mineral ${ }^{17}$. But this has to date been absent in artificial nacres ${ }^{19-24}$. Although recently few works have incorporated amorphous phases as reinforcement, such as amorphous alumina nanosheet efficiently bonding with polylactic acid to enhance the toughness of the material ${ }^{16}$, this is very different from the natural configuration of the amorphous/crystalline heterophase existing within the nacre platelets.

$\mathrm{MnO}_{2}$ is generally used as an excellent electrochemical material ${ }^{25}$. Recently, leaflike $\mathrm{MnO}_{2}$ hexagon nanosheets containing amorphous/crystalline structural feature were prepared, where $\sim 0.6 \mathrm{~nm}$ thick microporous mesophyll-like amorphous monolayer with higher oxygen vacancies crosslinks itself or other nanosheets and the 2-4 nm thick vein-like crystalline skeleton supports the whole structure ${ }^{25}$. We expect it to be an ideal reinforcer to composite with GO to create sophisticated amorphous/crystalline heterophase platelet. Based on this concept, we construct a centimetre-sized, $\mathrm{GO} / \mathrm{MnO}_{2}$-based layered (GML) bulk that replicates nacreous 
amorphous/crystalline heterophase platelets and embodies high-density crosslinking interfaces at multiscale interfaces. We accomplish this by assembling the microscale building blocks in a simple bottom-up assembly. These building blocks are composed of nanoscale amorphous/crystalline leaf-like $\mathrm{MnO}_{2}$ hexagon/graphene oxide (A/C$\mathrm{LMH} / \mathrm{GO}$ ) heterophase reinforcing units glued by polymer-based crosslinkers. The resultant GML bulk achieves higher flexural mechanical performance (strength, 194.7 $\pm 9.2 \mathrm{MPa}$; specific strength, $105.2 \pm 5.0 \mathrm{MPa} / \mathrm{Mg} \cdot \mathrm{m}^{-3}$ ) than nearly all of the other GObased bulk composites, excellent fracture toughness $\left(5.4 \pm 0.4 \mathrm{MPa} \mathrm{m}^{1 / 2}\right)$, and an attractive impact resistance $\left(51.5 \pm 9.2 \mathrm{MJ} \mathrm{m}^{-3}\right)$. The strengthening and toughening effects are also corroborated by multi-level simulations.

Design, assembly, and characterization. To imitate structural features of natural nacre (Supplementary Fig. 1), we design our GML bulk using a bottom-up assembly process (Fig. 1a,b) that featured the following five steps: i) the A/C-LMH nanosheet with excellent mechanical properties (the nanoindent Young's modulus $\left(E_{\mathrm{n}}\right)$ and hardness $(H)$ of the assembled A/C-LMH film are 80.3 $\pm 13.2 \mathrm{GPa}$ and $3.2 \pm 0.9 \mathrm{GPa}$, respectively.) as a reinforcing phase (see Methods and Supplementary Figs. 2 and 3), is inserted into the interlamination of $\mathrm{GO}$ nanosheets, forming a stronger $\mathrm{A} / \mathrm{C}-\mathrm{LMH} / \mathrm{GO}$ heterophase than the crystalline $\mathrm{MnO}_{2}$ homo-phase (Supplementary Figs. 4 and 5). ii) Both sodium alginate (SA) and regenerated silk fibroin (rSF) fibres as soft organic phases are used to mimic soft $\beta$-chitin fibril and analogous silk protein (Supplementary Figs. 6 and $7)^{22,24}$. These fibres can adsorb onto the surfaces and at the interfaces of the A/C$\mathrm{LMH} / \mathrm{GO}$ heterophase to further crosslink the heterophase interfaces via intermolecular forces (e.g., hydrogen bonds, van der Waals forces). iii) Borate ions, which lead to crosslinking in many vascular plant tissues ${ }^{26}$, are also incorporated into the critical interphase to further reinforce the interfacial crosslinking interactions among the different constituents by strong B-O covalent bonding (Supplementary Fig. 8), facilitating high-density interfacial formation. iv) The nanoscale structural units are further assembled to form a layered nanocomposite film used as a microscale building block by evaporation-assisted self-assembly (ESA) (Fig. 1a and Supplementary Fig. $9)^{14,24}$. v) These building blocks are further accumulated to construct a highly ordered, dense GML bulk through stacking-up and hot-press techniques, which enables us to greatly enhance multiscale interfacial crosslinking interactions. The detailed preparation process and characterization are as follows:

In the first stage, before incorporating the cross-linking additives (e.g., SA, rSF, and borate), the optimal weight ratios of $\mathrm{A} / \mathrm{C}-\mathrm{LMH}$ to $\mathrm{GO}$ were determined to be $1 / 3$ by tensile tests of binary $\mathrm{GO} / \mathrm{MnO}_{2}$ films. Next, different types of layered nanocomposite films were prepared from aqueous ternary, quaternary, and quinary suspensions by adjusting the incremental content of the additives described above (see Methods). Through tensile tests, the optimal weight percentage of the mechanically superior quaternary nanocomposite film (named $\mathrm{GO} / \mathrm{MnO}_{2} / \mathrm{SA} / \mathrm{rSF}$ ) was $74.4 \mathrm{wt} \% \mathrm{GO}$, $24.8 \mathrm{wt} \% \mathrm{MnO}_{2}, 0.5 \mathrm{wt} \% \mathrm{SA}$, and $0.3 \mathrm{wt} \% \mathrm{rSF}$ (74.4-24.8-0.5-0.3) (Supplementary Table 1). Fig. 2a shows these quaternary films with brown, flat, and regular features. Many A/C-LMH nanosheets are randomly embedded into the interlamination of GO 
nanosheets to establish the staggered stacking heterophase, and the orientation of A/CLMH nanosheets is preferentially parallel to that of GO nanosheets (Fig. 2b,c). Mappings of several key elements and the formation of the complex stacking of the 2D heterogeneous structure were confirmed by high-angle annular dark field scanning transmission electron microscopy (HAADF-STEM) imaging with energy dispersive spectrometry (EDS) (Fig. 2d,e). At a finer scale, the heteroplastic layers within the nanocomposite film remained relatively well-aligned and interlaced, as demonstrated by the small-angle X-ray scattering (SAXS) patterns and scanning electron microscopy (SEM) images (Fig. 2f,g and Supplementary Fig. 10). Simultaneously, the gallery spacing ( $d$-spacing) value was smaller than that of pure GO paper, indicating that a much more densely packed nanostructure greatly facilitated the formation of strong synergistic interfacial interactions among different compositions during their relative sliding. Additionally, the active sites of these additives bonded strongly with the oxygen-containing functional groups of GO by synergistic crosslinking interactions, as verified by a series of characterizations (Supplementary Figs. 11-15) ${ }^{27-31}$.

In the second stage, a number of quaternary nanocomposite films of equal size (6 $\mathrm{cm} \times 6 \mathrm{~cm})$ and ideal thickness $(\sim 20 \mu \mathrm{m})$ were glued together with a thin layer $(\sim 1 \mu \mathrm{m})$ of mixed borate/SA solution being brush-coated on the surface of each film to form laminates with a total of 180 layers (Supplementary Fig. 16a,b). The thin layer was expected to incorporate binary borate/SA crosslinking interactions at the interfaces between adjacent films by the combined action of hydrogen and covalent bonds. During assembly, the films were carefully aligned so that these building blocks formed a highly ordered 3D BM arrangement ${ }^{19}$. After further pre-compression and drying, the bulk material was subsequently soaked in a mixed borate/SA solution to further interconnect/fix the multiscale interfaces (including nanoscale A/C-LMH/GO interfaces and microscale film-film interfaces) by the in-situ crosslinking SA with borate ions. Next, a hot-pressing process was used to further build a uniform orientation of the assembling lamellas and improve the synergetic crosslinking interactions. Finally, a brown GML bulk with a width of $5.0 \mathrm{~cm} \times 5.0 \mathrm{~cm}$, a thickness of $3.0 \mathrm{~mm}$, and a density of $1.85 \mathrm{~g} \mathrm{~cm}^{-3}$ was successfully prepared, as shown in Fig. 2h. Its constituent content was approximately $69.4 \mathrm{wt} \% \mathrm{GO}, 23.3 \mathrm{wt} \% \mathrm{MnO}_{2}, 6.5 \mathrm{wt} \% \mathrm{SA}, 0.5 \mathrm{wt} \% \mathrm{rSF}$, and $0.3 \mathrm{wt} \%$ borate (Supplementary Tables 2 and 3). The resulting densely layered structure at the fracture surface and the cross-section can be observed in Fig. $2 \mathrm{i}$ and Supplementary Fig. 17, suggesting that the relatively isolated interfacial reinforcing units in adjacent films were interconnected with each other by bridging compositions, which were strikingly similar to those of natural C. plicata nacre (Fig. 2j). For comparison, we also prepared three other kinds of representative 3D specimens, including pure GO bulk, pure SA bulk, and non-borate/SA-immersed samples (Supplementary Fig. 16c,d).

Analysis of mechanical properties. To test the validity of the nanoscale heterophase reinforcing structural unit, the mechanical responses of these nanocomposite films were studied, as shown in Fig. 3a-h. The binary nanocomposite film $\left(\mathrm{GO} / \mathrm{MnO}_{2}\right)$ displays a better micromechanical performance than pure GO and other binary competitors (such 
as $\mathrm{GO} / \mathrm{rSF}$ and $\mathrm{GO} / \mathrm{SA}$, Fig. $3 \mathrm{~b}, \mathrm{c}) . E_{\mathrm{n}}$ and $H$ of $\mathrm{GO} / \mathrm{MnO}_{2}$ were measured to be $13.5 \pm$ $1.5 \mathrm{GPa}$ and $1.6 \pm 0.2 \mathrm{GPa}$, which are approximately $83.9 \%$ and $100.0 \%$ higher than those of the pure GO film. The values in $E_{\mathrm{n}}$ and $H$ for the optimal quaternary nanocomposite film $\left(\mathrm{GO} / \mathrm{MnO}_{2} / \mathrm{rSF} / \mathrm{SA}\right)$ are $16.3 \pm 2.0 \mathrm{GPa}$ and $1.4 \pm 0.2 \mathrm{GPa}$, respectively, which are clearly superior to those of the unary film and higher than those of other nanocomposite films (Fig. 3a-c, Supplementary Fig. 18). The tensile tests further reveal that $\mathrm{GO} / \mathrm{MnO}_{2}$ achieves better tensile mechanical properties than pure $\mathrm{GO}$ and crystalline/crystalline homo-phase structural $\mathrm{GO} / \mathrm{MnO}_{2}$ (Supplementary Table 1). Furthermore, the tensile mechanical properties of $\mathrm{GO} / \mathrm{MnO}_{2} / \mathrm{rSF} / \mathrm{SA}$, due to their unique integration of the highest tensile strength $\left(\sigma_{\mathrm{u}}, 368.1 \pm 9.9 \mathrm{MPa}\right)$ and impressive work of toughness $\left(W, 13.8 \pm 2.6 \mathrm{MJ} \mathrm{m}^{-3}\right)$, are superior to those of the other nanocomposite films (Fig. 3d-f). Compared with unary films, the maximum Young's modulus $\left(E_{\mathrm{m}}\right)$ of $\mathrm{GO} / \mathrm{MnO}_{2} / \mathrm{rSF} / \mathrm{SA}$ is clearly enhanced, up to $9.5 \pm 1.5 \mathrm{GPa}$ (Fig. 3f). We confirmed that, combined with the fracture cross-sectional observation of typical nanocomposite films (Supplementary Fig. 19), A/C-LMH nanosheets could effectively bond with $\mathrm{GO}$ nanosheets to form a stronger $\mathrm{A} / \mathrm{C}-\mathrm{LMH} / \mathrm{GO}$ reinforcing interface; the polymer-based cross-linking interactions are conducive to further enhance the loadbearing capability of the $\mathrm{A} / \mathrm{C}-\mathrm{LMH} / \mathrm{GO}$ reinforcing unit. Thus, the optimal quaternary nanocomposite film achieved an exceptional mechanical combination and constituted an ideal building block for the GML bulk assembly.

To further estimate the effectiveness of the highly ordered heterophase structure at multiscale interfaces, the mechanical response of the as-fabricated GML bulk was systematically studied, as shown in Fig. 4. Its flexural mechanical properties were first investigated by three-point bending tests to compare with those of other bulk materials, such as pure GO bulk, SA bulk, and non-borate/SA-immersed bulk materials (Fig. 4ac, Supplementary Fig. 20), respectively. The flexural mechanical properties of the GML bulk were clearly higher than those of other bulk materials. The flexural strength of the GML bulk was $194.7 \pm 9.2 \mathrm{MPa}$ (the specific strength is up to $105.2 \pm 5.0 \mathrm{MPa} / \mathrm{Mg} \cdot \mathrm{m}^{-}$ ${ }^{3}$ ), which is eleven times higher than that of the pure GO bulk $(17.4 \pm 3.4 \mathrm{MPa})$ and two times higher than that of pure SA bulk $(78.5 \pm 16.2 \mathrm{MPa})$ and the non-borate/SAimmersed bulk $(72.3 \pm 3.1 \mathrm{MPa})$. Even more noteworthy is that our GML bulk represents the highest yield strength among GO-based bulk nanocomposites reported to date ${ }^{10,12,13}$. A joint improvement in the strength $(194.7 \pm 9.2 \mathrm{MPa})$ and stiffness (7.0 $\pm 0.2 \mathrm{GPa})$ is depicted in Fig. $4 \mathrm{~b}$ after the laminations of those quaternary films were mediated by the mixed borate/SA networks. Furthermore, the fracture toughness for crack initiation, $K_{\mathrm{Ic}}$, as an evaluation of the resistance to crack initiation of the GML bulk $\left(0.87 \pm 0.03 \mathrm{MPa} \cdot \mathrm{m}^{1 / 2}\right)$ is lower than that of natural nacre $\left(3-10 \mathrm{MPa} \mathrm{m}^{1 / 2}\right)^{18}$, but higher than that of pure GO bulk $\left(0.13 \pm 0.04 \mathrm{MPa} \cdot \mathrm{m}^{1 / 2}\right)$, SA bulk $\left(0.5 \pm 0.04 \mathrm{MPa} \cdot \mathrm{m}^{1 / 2}\right)$, and the no borate/SA immersing specimen $\left(0.22 \pm 0.02 \mathrm{MPa} \cdot \mathrm{m}^{1 / 2}\right)$. However, the fracture toughness for stable crack propagation of the GML bulk, $K_{\mathrm{Jc}}(5.4 \pm 0.4 \mathrm{MPa}$ $\left.\mathrm{m}^{1 / 2}\right)$, is comparable to that of the natural C. Plicata nacre $\left(\sim 5.9 \mathrm{MPa} \mathrm{m}^{1 / 2}\right)$ and exceeds that of the disordered brushite-SA composite $\left(\sim 3.8 \mathrm{MPa} \mathrm{m}^{1 / 2}\right)^{24}$, as shown in Fig. 4c.

The indentation impressions on the GML bulk were further investigated with SEM and optical microscopy (Fig. 4d, Supplementary Figs. 21 and 22). The results were 
compared with the geologic GO bulk to further estimate the resistance to damage localization. An indentation load $(800 \mathrm{mN}$ peak load) was applied to both the free surface layer of the GO bulk and GML bulk. The damage area/size $\left(A_{\mathrm{d}}=9503.3 \mu \mathrm{m}^{2}\right.$, $\left.r_{\mathrm{d}}=\sim 55 \mu \mathrm{m}\right)$ in the layer of the GML bulk was slightly smaller than that of GO $\left(A_{\mathrm{d}}=\right.$ $\left.16286.0 \mu \mathrm{m}^{2}, r_{\mathrm{d}}=\sim 72 \mu \mathrm{m}\right)$. For the GML bulk, small cracks or external damages were observed in the post-indentations, which also correlated with their indentation curves (Supplementary Fig. 22). Inhibition or delay cracking at sharp contacts in some biomaterials can be due to the capabilities of these materials to endure localized shear deformation ${ }^{32}$. This mechanism results in the contact stress redistribution under the contact point, generally giving rise to the formation of a significant residual stress field. To measure this mechanism applied to our 3D bulk material, the shifts in the D band of GO around the indents in these samples were detected by using high-resolution Raman microspectroscopy (Fig. 4d, middle), which can be used to estimate the residual stress raised in the same area ${ }^{32}$. The residual stress field in the GML bulk was almost three times larger than in GO bulk (Fig. 4d, right). This result further demonstrates that a high fraction of indentation energy is released by cracking in the geologic GO, thus leaving a small residual stress field. A larger yielding zone developed around the contact point in the GML bulk, establishing a complex residual stress field where a large quantity of contact energy is absorbed. Similarly, the in-situ observation of the 3D bulk specimen under single-edge notched bending tests also shows that the main crack propagated along a largely tortuous path at the interfaces of these stacked laminates (Fig. 4e, Supplementary Fig. 23). The large crack deflection, branching, and multiple cracks at the crack tips (Fig. 4f,g) can all be recognized as effective jointly intrinsic and extrinsic toughening mechanisms, which can decrease the crack deriving force, similar to that of natural structural materials ${ }^{32,33}$.

Further molecular dynamics (MD) and finite element (FE) simulations quantitatively verified the above strengthening and toughening mechanisms, as shown in Fig. 5a-d, Supplementary Figs. 24-27 and Tables 4,5, and Movies S1-S3. In the molecular model, MD simulation corroborated that the amorphous- $\mathrm{MnO}_{2} / \mathrm{GO}$ interface strength was much stronger than the crystalline- $\mathrm{MnO}_{2} / \mathrm{GO}$ interface strength (Fig. 5a,b), suggesting the stronger binding capability of amorphous- $\mathrm{MnO}_{2}$ and GO. In the nanoscale model, FE simulation verified that a typical crack propagated along the heterogeneous interface path (Fig. 5c, upper), and further interface failure was dominated by interface sliding with the friction, plasticity, and nanosheet pull-out. Notably, the strength of the optimized heterophase reinforcing interface (approximately $30 \mathrm{MPa}$ ) play a key role in achieving an ideal flexural strength (about $200 \mathrm{MPa}$ ). In the microscale model (Fig. 5c, bottom), FE simulation further proved that a distinct crack deflected along the interlayer between lamellae, and the further failure of the interlayer is mainly dominated by interlayer sliding with friction, deformation, and lamellar peeloff, accompanied by multiple second microcrack nucleation and branching, similar to those observed in the GML bulk (Figs. 4e-g, Supplementary Fig. 23). Clearly, the integration of the optimized interlayer strength and the excellent lamellar strength efficiently insures similar flexural mechanical properties of the GML bulk (Fig. 5d), where the lamellar strength is very close to that of our optimized nanocomposite film 
(Fig. 2d). Therefore, this highly ordered heterophase platelets combined with multiscale interface crosslinking is mainly responsible for excellent mechanical responses.

From the above analysis, we considered that, the amorphous/crystalline reinforcing unit combined with polymer-based crosslinkers at molecular-nanoscale levels, could offer an intrinsic toughening mechanism, enhancing the initial mechanical properties of the nanocomposite film, whereas the microscale lamellar structure produces extrinsic toughening effects, improving energy dissipation during fracture (since when the crack encounters the complex inorganic-organic interfaces within lamellas, the adhered layers can provide efficient energy dissipation ${ }^{33-36}$ ).

Impact resistance and mechanical performance comparison. This strong and tough yet lightweight 3D bulk holds promise as a material for excellent armour and impact energy absorption. To prove such a potential, split Hopkinson pressure bar (SHPB) experiments were carried out, as shown in Fig. 5e and Supplementary Fig. 28. The impact resistance ability of the GML bulk is stronger than that of other bulk materials. For example, the compression stress of the GML bulk can reach $\sim 240.0, \sim 187.0$, and $\sim 120.0 \mathrm{MPa}$, and the energy absorption of compression is $\sim 51.5, \sim 36.4$, and $\sim 23.0 \mathrm{MJ}$ $\mathrm{m}^{-3}$ under derive pressures of 55000,45000 , and $35000 \mathrm{~Pa}$, respectively. Under a drive pressure of $55000 \mathrm{~Pa}$, the value $\left(\sim 51.5 \mathrm{MJ} \mathrm{m}^{-3}\right)$ in the impact resistance of the GML bulk is more than four times that of the pure GO bulk material and more than two times that of pure SA and the no borate/SA immersing specimen. The stress-strain curves of the GML bulk reflect a higher impact modulus during the whole impact strain process than those of other bulk materials. These curves also illustrate a larger wave-like increase compared with that of the non-borate/SA-immersed bulk and are distinctly distinguished from the quasi-linear increase in the pure GO bulk and the large plastic platform area of the pure SA bulk. The above results directly demonstrate the excellent impact energy absorption properties of our GML bulk that suggest potential applications as an armoured material.

Fig. $5 \mathrm{f}$ shows that the specific toughness $\left(K_{\mathrm{c}} / \rho\right)$ and specific strength $\left(s_{f}\right)$ of the GML bulk were higher than those of a wide range of polymer engineering materials (e.g., poly(methyl methacrylate) (PMMA), polystyrene (PS), and polyurethane (PU).), as well as many natural nacre-inspired bulk materials (e.g., clay/polyvinyl acetate $(\mathrm{PVA})^{37}, \mathrm{Al}_{2} \mathrm{O}_{3} / \mathrm{GO}^{38}, \mathrm{Al}_{2} \mathrm{O}_{3} /$ polyetherimide (PEI) ${ }^{39}$ (see Supplementary Table 6), and some natural materials ${ }^{40}$. The GML bulk specific toughness and strength were also comparable to those of natural nacre ${ }^{18,19}$ and other unique bulk composites such as $\mathrm{MTM} / \mathrm{PVA} / \mathrm{resol}^{14}$. Owing to the excellent mechanical performance-especially its impressive (specific) flexural strength, resistance to impact, light weight, large size, and mild preparation processes - we envision widespread structural and functional applications for the material in the future.

\section{Conclusion}

By designing the appropriate amorphous/crystalline heterophase platelets and multiscale interface crosslinking interactions, we prepare a centimetre-sized, high- 
performance GML bulk, especially with an outstanding flexible strength, that is the strongest among reported GO-based bulk materials. Such performance can be originated from the regulating the balance of different interfacial interactions. First, the A/C-LMH nanosheet effectively bonds with GO nanosheet to form a strong heterophase interfacial interaction at molecular level, endowing the heterophase structural unit with intrinsic high strength, enhanced stiffness, and toughness. Second, the polymer-based crosslinkers further reinforce the nanoscale A/C-LMH/GO interfacial interactions, which can absorb larger amounts of mechanical energy to hinder crack initiation when loaded. Third, optimizing microscale film-film interfaces glued by the mixed crosslinkers enhances the microscale load transferring capability by larger crack deflection and interlayer shearing, increasing mechanical energy absorption and providing the material with extrinsic toughness, excellent impact resistance, and damage tolerance. GO-based composites are widely applicable and highly scalable for industrial use. We expect that the concept of heterophase platelets constructing biomimetic 3D bulk composites as well as the material developed in this work throw insights into the design of engineered structural materials and allow widespread use in aerospace, biomedicine and military applications.

\section{Methods}

Fabrication of GML bulk and no borate/SA immersing bulk material. To assemble the GML bulk, the prepared $\mathrm{GO} / \mathrm{MnO}_{2} / \mathrm{rSF} / \mathrm{SA}$ nanocomposite films were cut into pieces of equal sizes $(6.0 \mathrm{~cm} \times 6.0 \mathrm{~cm})$ and brush-coated with a thin layer $(h, \sim 1.0 \mu \mathrm{m})$ of SA solution on the surface. These films were then laminated together by stacking to form laminates with a total of 180 layers; the laminates were rolled using a glass rod, and transferred into a homemade aluminium alloy mould (see Supplementary Fig. 16a). Then, a pre-hot-pressing step with a pressure of $15 \mathrm{MPa}$ was applied at $40{ }^{\circ} \mathrm{C}$ for $\sim 24$ $\mathrm{h}$ to attain the "non-borate/SA-immersed bulk material". After that, this bulk material was immersed in a mixed borate/SA solution $(0.3 \mathrm{wt} \% \mathrm{SA}$ and $10 \mathrm{mg} / \mathrm{ml}$ borate) for 2 h. Finally, a hot-pressing step with a pressure of $25 \mathrm{MPa}$ at $50{ }^{\circ} \mathrm{C}$ for $\sim 48 \mathrm{~h}$ was used to obtain the GML bulk with a thickness of approximately $3.0 \mathrm{~mm}$.

Tensile test and nanoindentation of $2 \mathrm{D}$ nanocomposite films. The tensile mechanical properties were measured using a Shimadzu AGS-X Tester with a dynamic mechanical analyser under natural environmental conditions of $25^{\circ} \mathrm{C}$ and $30 \%$ relative humidity (RH). Static/cyclic tensile tests were evaluated at a load speed of $1 \mathrm{~mm} \cdot \mathrm{min}^{-1}$ with a gauge length of $5 \mathrm{~mm}$. All of the papers for the tensile test were cut into strips with a length of $20 \mathrm{~mm}$ and a width of $3 \mathrm{~mm}$. The specimen thickness was obtained from SEM imaging of the fracture edges. Micromechanical properties and surface features were measured under ambient conditions $\left(25^{\circ} \mathrm{C}, 30 \%\right.$ of $\left.\mathrm{RH}\right)$ by a commercial TI 950 triboindenter (Hysitron) equipped with a Berkovich diamond tip $(R \approx 100 \mathrm{~nm}$ ) using a continuous depth-sensing indentation technique. 
Flexural strength and strain of bulk materials. Three-point bending tests (Shimadzu AGS-X Tester) were performed on the bulk sample. A support span of $7.5 \mathrm{~mm}$ and a bending displacement rate of $2 \mu \mathrm{m} \mathrm{s}^{-1}$ were used in the three-point bending tests. The in-situ three-point bending tests of the bulk sample were performed in a FEI Quanta $250 \mathrm{FEG}$ environmental scanning electron microscope at $25^{\circ} \mathrm{C}$ using a Deben microtest tensile stage; loading was applied under displacement control at a displacement rate of $0.02 \mathrm{~mm} \mathrm{~min}^{-1}$.

(1) Calculation of stress $\sigma$ and strain $\varepsilon$

According to ASTM D790-03, stress and strain were calculated from the three-point bending force-displacement curves as follows ${ }^{14,24}$ :

$$
\begin{aligned}
& \sigma=\frac{3 F S}{2 b d^{2}} \\
& \varepsilon=\frac{6 D d}{\mathrm{~S}^{2}}
\end{aligned}
$$

where $F$ is the force, $D$ is the displacement, and $S$ is the length of support span. $b$ and $d$ represent the specimen width and thickness, respectively. Fracture strength and fracture strain are calculated using the $F$ and $D$ at failure.

(2) Measurement of Young's modulus

The Young's modulus, $E$, is obtained from the slope of the linear portion of the threepoint bending stress-strain curves.

Mechanical calculation of three-point bending tests. Fracture toughness for crack initiation, $K_{\text {Ic }}$, is estimated by the following equation ${ }^{24}$ :

$$
\begin{gathered}
K_{\mathrm{Ic}}=\frac{P_{\mathrm{Ic}} S}{B W^{\frac{3}{2}}} f\left(\frac{a}{W}\right) \\
f\left(\frac{a}{W}\right)=\frac{3\left(\frac{a}{W}\right)^{1 / 2}\left[1.99-\left(\frac{a}{W}\right)\left(1-\frac{a}{W}\right)\left(2.15-\frac{3.93 \mathrm{a}}{W}+\left(\frac{a}{W}\right)^{2}\right)\right]}{2\left(1+\frac{2 a}{W}\right)\left(1-\frac{a}{W}\right)^{3 / 2}}
\end{gathered}
$$

where $P_{\text {Ic }}$ is the maximum load in the single-edge notched bend tests, $S$ is the support span, $B$ is the thickness of the specimen, $W$ is the width of the specimen, and $a$ is the notch depth. Fracture toughness, $K_{\mathrm{Jc}}$, in this study is calculated from the elastic and plastic contribution, which refers to $J$-integral calculation, consistent with previously reported methods for estimating the properties of bone and other composites ${ }^{21,35}$.

$$
J=J_{\mathrm{el}}+J_{\mathrm{pl}}
$$

$J_{\mathrm{el}}$ is the elastic contribution, which is on the basis of linear elastic fracture mechanics.

$$
J_{e l}=\frac{K_{\mathrm{IC}}^{2}}{E^{\prime}}
$$

The plastic contribution $J_{\mathrm{pl}}$ can be calculated with the following equation:

$$
J_{\mathrm{pl}}=\frac{2 A_{\mathrm{pl}}}{B(W-a)}
$$

where $A_{\mathrm{pl}}$ is the plastic area underneath the load-displacement curve. So, $J$ values can be transformed into $K$ values by the following equation:

$$
K_{\mathrm{JC}}=\left(J E^{\prime}\right)^{1 / 2}
$$

in which, $E^{\prime}=E\left(1-v^{2}\right), E$ is Young's modulus, and $v$ is the Poisson's ratio. Since the variation of $E$ influences $K_{\mathrm{JC}}$ in a fairly limited way, here, $E^{\prime}$ can be replaced by $E$. 


\section{Acknowledgements}

We acknowledge the funding support from the National Natural Science Foundation of China (51532001 and 21905011) and China Postdoctoral Science Foundation (2017M610030).

\section{Author contributions}

L.G., K.C., X.T., B.J. C.C., L.D., and Y.W. conceived the project and designed the experiments. K.C., X.T., and B.J. carried out the design and fabrication of the lamellar composites. X.T., K.C., and B.J. characterized all the samples. X.T., J.H, and K.C. performed the mechanical testing. K.C., X.T., and J.H performed in-situ tensile tests. L.D. and C.C. carried out FE simulation. X.T. and C.C. made the Movies. L.G., K.C., X.T., L.D., C.C., Y.W., B.J., T. X., and K. G. wrote the manuscript. All the authors discussed the results and commented on the manuscript.

\section{Corresponding author}

Email: guolin@buaa.edu.cn, fax \& Tel: +86-010-82338162.

\section{Competing interests}

The authors declare no competing financial interest.

\section{Additional information}

Supplementary information is available free of charge on the .......website at DOI:

\section{Data availability}

The authors declare that all data supporting the findings of this study are available within the paper and its Supplementary Information. Other supporting data are available from the corresponding author upon request.

\section{Author information}

\section{ORCID}

Ke Chen: 0000-0001-7612-0226

Lin Guo: 0000-0002-6070-2384

\section{References}

1. Dikin, D. A., et al. Preparation and characterization of graphene oxide paper. Nature 448, 457-460 (2007).

2. Bai, H., Li, C. \& Shi, G. Functional composite materials based on chemically converted graphene. Adv. Mater. 23, 1089-1115 (2011).

3. Joshi, R. K., Carbone, P., Wang, F. C., Kravets, V. G., Su, Y., Grigorieva, I. V., Wu, H. A., Geim, A. K. \& Nair, R. R. Precise and ultrafast molecular sieving through graphene oxide membranes. Science 343, 752-754 (2014).

4. Yeh, C.-N., Huang, H., Lim, A. T. O., Jhang, R.-H., Chen, C.-H. \& Huang, J. X. Binder-free graphene oxide doughs. Nat. Commun. 10, 422 (2019). 
5. Xin, G., Yao, T., Sun, H., Scott, S. M., Shao, D., Wang, G. \& Lian, J. High thermally conductive and mechanically strong graphene fibers. Science 349, 1083-1087 (2015).

6. Chang, D., et al. Reversible fusion and fission of graphene oxide-based fibers. Science 372, 614-617 (2021).

7. Hu, K., Gupta, M. K., Kulkarni, D. D. \& Tsukruk, V. V. Ultra-robust graphene oxidesilk fibroin nanocomposite membranes. Adv. Mater. 25, 2301-2307 (2013).

8. Zhao, C., et al, Layered nanocomposites by shear-flow-induced alignment of nanosheets. Nature 580, 210-215 (2020).

9. An, Z., Compton, O. C., Putz, K. W., Brinson, L. C., Brinson, C. \& Nguyen, S. T. Bio-inspired borate cross-linking in ultra-stiff graphene oxide thin films. Adv. Mater. 23, 3842-3846 (2011).

10. Wang, C., Chen, X., Wang, B., Huang, M., Wang, B., Jiang, Y. \& Ruoff, R. S. Freeze-casting produces a graphene oxide aerogel with a radial and centrosymmetric structure. ACS Nano 12, 5816-5825 (2018).

11. Zhu, C., Han, Y.-J., Buoss, E. B., Golobic, A. M., Kuntz, J. D., Spadaccini, C. M. \& Worsley, M. A. Highly compressible 3D periodic graphene aerogel microlattices. Nat. Commun. 6, 6926 (2015).

12. Wang, B., et al. Folding large graphene-on-polymer films yields laminated composites with enhanced mechanical performance. Adv. Mater. 30, 1707449 (2018).

13. Jia, H., Li, Y., Luan, Y., Zheng, Y., Yang, J., Wang, L., Guo, Z. \& Wu, X. Bioinspired nacre-like GO-based bulk with easy scale-up process and outstanding mechanical properties. Compos. Part A 132, 105829 (2020).

14. Chen, S. M., et al. Superior biomimetic nacreous bulk nanocomposites by a multiscale soft-rigid dual-network interfacial design strategy. Matter 1, 412-427 (2019).

15. Meyers, M. A., McKittrick, J. \& Chen, P. Y. Structural biological materials: critical mechanics-materials connections. Science 339, 773-779 (2013).

16. Chen, K., Ding, J., Li, L., Shang, G., Yue, Y. \& Guo, L. Amorphous alumina nanosheets/polylactic acid artificial nacre. Matter 1, 1385-1398 (2019).

17. Nassif, N., Pinna, N., Gehrke, N., Antonietti, M., Jäger, C. \& Cölfen, H. Amorphous layer around aragonite platelets in nacre. Proc. Natl. Acad. Sci. USA. 102, 1265312655 (2005).

18. Wegst, U. G. K., Bai, H., Saiz, E., Tomsia, A. P., \& Ritchie, R. O. Bioinspired structural materials. Nat. Mater. 14, 23-26 (2015).

19. Wang, J., Cheng, Q., \& Tang, Z. Layered nanocomposites inspired by the structure and mechanical properties of nacre. Chem. Soc. Rev. 41, 1111-1129 (2012).

20. Yin, Z., Hannard, F. \& Barthelat, F. Impact-resistant nacre-like transparent materials. Science 364, 1260-1263 (2019).

21. Bouville, F., et al. Strong, tough and stiff bioinspired ceramics from brittle constituents. Nat. Mater. 13, 508-514 (2014).

22. Mao, L. B., et al. Synthetic nacre by predesigned matrix-directed mineralization. Science 354, 107-110 (2016). 
23. Cantaert, B., Kuo, D., Matsumura, S., Nishimura, T., Sakamoto, T. \& Kato, T. Use of amorphous calcium carbonate for the design of new materials. ChemPlusChem 82, 107-120 (2017).

24. Gao, H. L., et al. Mass production of bulk artificial nacre with excellent mechanical properties. Nat. Commun. 8, 287 (2017).

25. Jia, B., Chen, W., Luo, J., Yang, Z., Li, L. \& Guo, L. Construction of $\mathrm{MnO}_{2}$ artificial leaf with atomic thickness as highly stable battery anodes. Adv. Mater. 32, 1906582 (2019).

26. O’Neill, M. A., Eberhard, S., Albersheim, P. \& Darvill A. G. Requirement of borate cross-linking of cell wall rhamnogalacturonan II for arabidopsis growth. Science 294, 846-849 (2001).

27. Lambert, J. B., Shurvell, H. F., Lightner, D. A. \& Cooks, R. G. Organic Structural Spectroscopy. 2 ed.; Prentice Hall: New York, 2001.

28. Ni, H., et al. Robust bioinspired graphene film via $\pi-\pi$ cross-linking. ACS Appl. Mater. Inter. 9, 24987-24992 (2017).

29. Chen, K., et al. Bioinspired interfacial chelating-like reinforcement strategy toward mechanically enhanced lamellar materials. ACS Nano 12, 4269-4279 (2018).

30. Park, S., et al. Graphene oxide papers modified by divalent ions-enhancing mechanical properties via chemical cross-linking. ACS Nano 2, 572-578 (2008).

31. Dong, X., Zhao, Q., Xiao, L., Lu, Q. \& Kaplan, D. L. Amorphous silk nanofiber solutions for fabricating silk-based functional materials. Biomacromolecules 17, 3000-3006 (2006).

32. Amini, S., Tadayon, M., Idapalapati, S. \& Miserez, A. The role of quasi-plasticity in the extreme contact damage tolerance of the stomatopod dactyl club. Nat. Mater. 14, 943-950 (2014).

33. Li, L. \& Ortiz, C. Pervasive nanoscale deformation twinning as a catalyst for efficient energy dissipation in a bioceramic armour. Nat. Mater. 13, 501-507 (2014).

34. Yeom, B., et al. Abiotic tooth enamel. Nature 543, 95-98 (2017).

35. Koester, K. J. \& Ritchie, R. O. The true toughness of human cortical bone measured with realistically short cracks. Nat. Mater. 7, 672-677 (2008).

36. Kolednik, O., Predan, J., Fischer, F. D. \& Fratzl, P. Bioinspired design criteria for damage-resistant materials with periodically varying microstructure. Adv. Funct. Mater. 21, 3634-3641 (2011).

37. Morits, M., et al. Toughness and fracture properties in nacre-mimetic clay/polymer nanocomposites. Adv. Funct. Mater. 27, 1605378 (2017).

38. Wang, L., et al. Microstructure and mechanical properties of nacre-like alumina toughened by graphene oxide. Ceram. Int. 45, 8081-8086 (2019).

39. Grossman, M., et al. Hierarchical toughening of nacre-like composites. Adv. Funct. Mater. 29, 1806800 (2019).

40. Wegst, U. G. K. \& Ashby, M. F. The mechanical efficiency of natural materials. Philos. Mag. 84, 2167-2186 (2007). 


\section{Figure Legends}

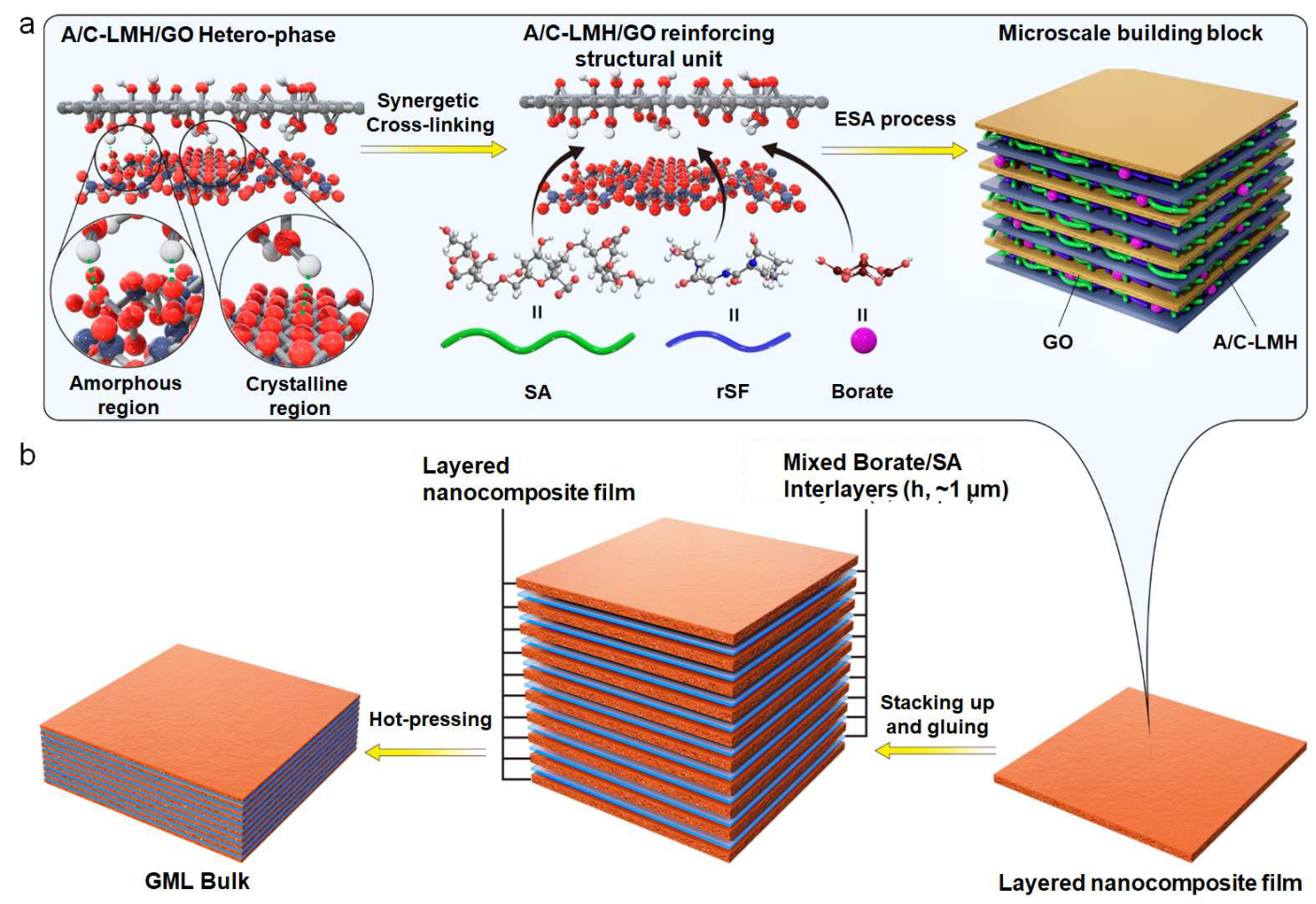

Fig. 1 I Schematic illustration for the design and assembly of the GML bulk material featuring amorphous/crystalline heterophase platelets and multiscale crosslinked interfaces. a, A/C-LMH nanosheet deposits on the surface of GO nanosheet, forming nanoscale $\mathrm{A} / \mathrm{C}-\mathrm{LMH} / \mathrm{GO}$ hetero-phase by hydrogen bonds at amorphous and crystalline regions. Subsequently, some additives (such as SA, rSF, and/or borate) incorporate into the surface and interface of the A/C-LMH/GO heterophase, forming a nanoscale $\mathrm{A} / \mathrm{C}-\mathrm{LMH} / \mathrm{GO}$ reinforcing unit by the in-situ complex cross-linking, which can further extend to the overall nanocomposite film as a microscale building block. $\mathbf{b}$, These thin mixed borate/SA crosslinking interlayers in the adjacent nanocomposite films can be further interconnected into the macroscopic 3D multi-scale crosslinking network in a stacking-up and hot-pressing process for assembling the GML bulk. Integration of the molecular scale A/C-LMH/GO heterophase, the nanoscale crosslinking interfaces and the microscale film-film interfaces can be precisely optimized by controlling the content of different constituents and assembly technology. 

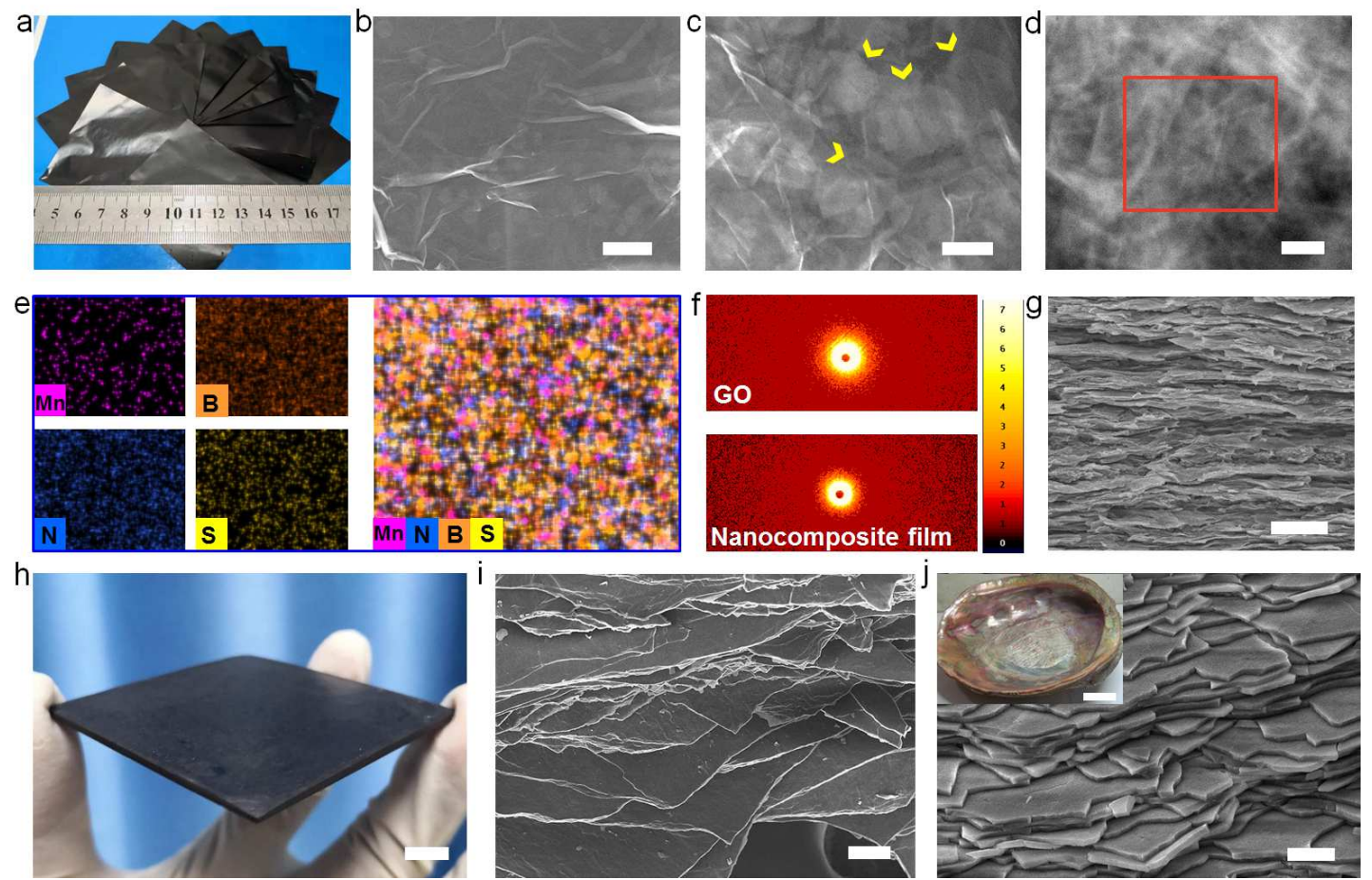

Fig. 2 | Structural and component characterization of the as-fabricated typical nanocomposite films and GML bulk. a, Photograph of the cut species from large-area quaternary nanocomposite film. b,c, The large-area SEM (b) and TEM (c) images depicting the uniformly scattered $\mathrm{A} / \mathrm{C}-\mathrm{LMH}$ nanosheets laid in the interlamination of the GO nanosheets to form the $\mathrm{A} / \mathrm{C}-\mathrm{LMH} / \mathrm{GO}$ hetero-phase within the nanocomposite film. Scale bars: $1 \mu \mathrm{m}(\mathbf{b})$ and $200 \mathrm{~nm}(\mathbf{c})$. Yellow arrows indicate the A/C-LMH nanosheets. d,e, The HAADF-STEM image (d) and corresponding EDS elemental maps (e) of the 2D entangled stacking heterogeneous structural phase, showing several key elements such as Mn, N, B, and S originating from different constituents in quinary nanocomposite film, selected red rectangular region in $\mathbf{d}$, revealing the even distribution of A/C-LMH nanosheets and additives. Scale bar: $200 \mathrm{~nm}$. f,g, The SAXS pattern (f) and the cross-section morphology (g) exhibiting an interlaced layered arrangement in nanocomposite film such as $\mathrm{GO} / \mathrm{MnO}_{2} / \mathrm{SA} / \mathrm{rSF}$. Scale bar: $500 \mathrm{~nm}$. h, Snapshot of the as-fabricated GML bulk. Scale bar: $1 \mathrm{~cm}$. i,j The cross-section morphologies of the fracture surfaces of the resultant GML bulk (i) and natural nacre (j) from abalone shell (j, inset), indicating the similar lamellar microstructure. Scale bars: $50 \mu \mathrm{m}(\mathbf{i}), 3 \mu \mathrm{m}(\mathbf{j})$ and $1 \mathrm{~cm}(\mathbf{j}$, inset). 

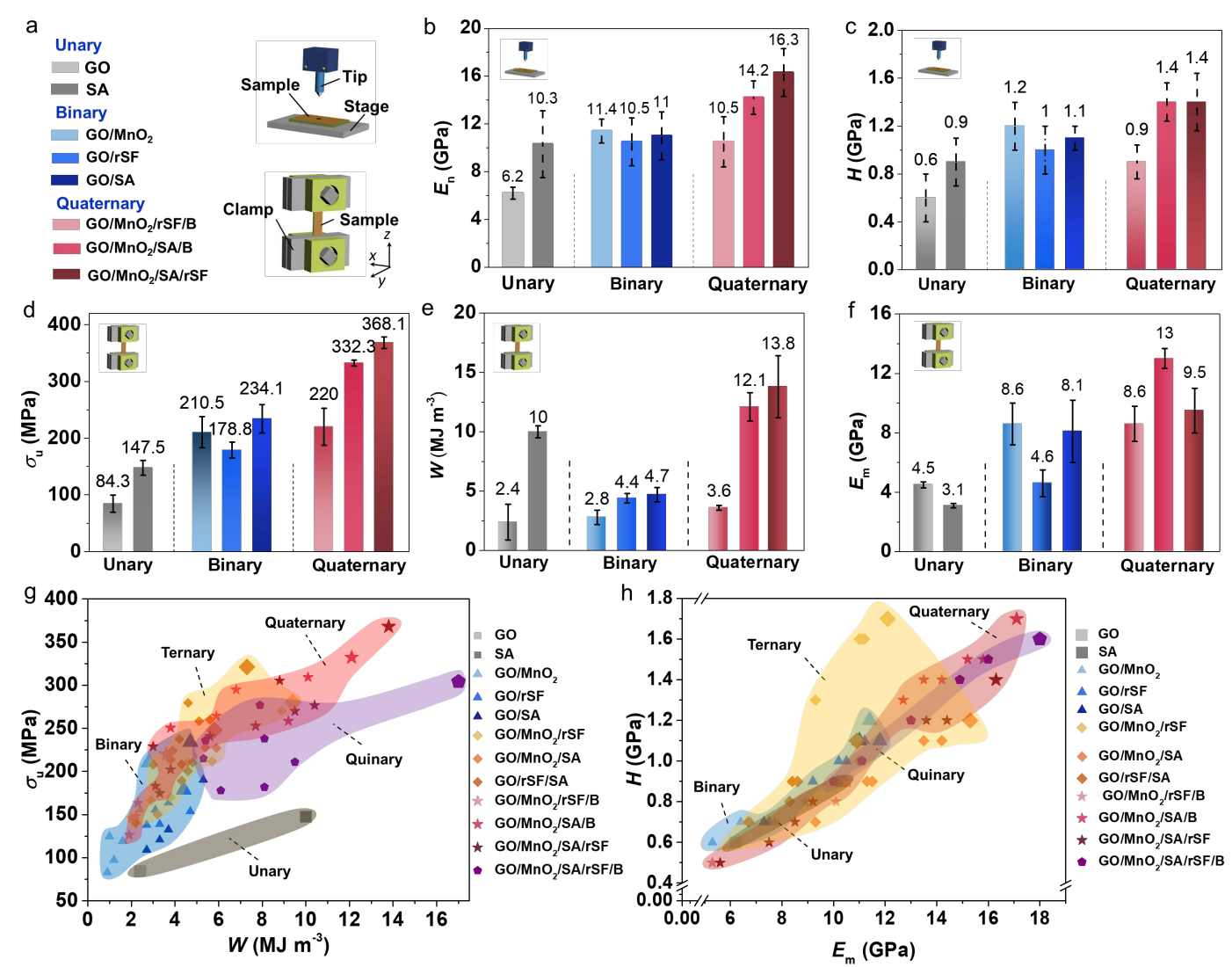

Fig. 3 | Mechanical properties of the as-fabricated layered nanocomposite films.

a, Sample preparation scheme for one-direction $(z)$ probing of nanoindentation contact responses and uniaxial tensile tests. Note that the color coding represents the different types of samples. b,c, Partial loading-unloading nanoindentation tests for graphical comparison of Young's modulus $\left(E_{\mathrm{n}}\right)(\mathbf{b})$ and hardness $(H)$ (c) under the force control mode (nearly $3000 \mu \mathrm{N}$ ). d-f, Uniaxial tensile tests for graphical comparison of the ultimate stress $\left(\sigma_{\mathrm{u}}\right)(\mathbf{d})$, the work of toughness $(W)(\mathbf{e})$, and the maximum Young's modulus $\left(E_{\mathrm{m}}\right)(\mathbf{f})$. The error bars are the standard deviations of the measured properties, $n=5$ for each specimen. $\mathbf{g}$, Comparison of $\sigma_{\mathrm{u}}$ and $W$ of these nanocomposite films. $\mathbf{h}$, Comparison of $H$ and $E_{m}$ of these nanocomposite films. 

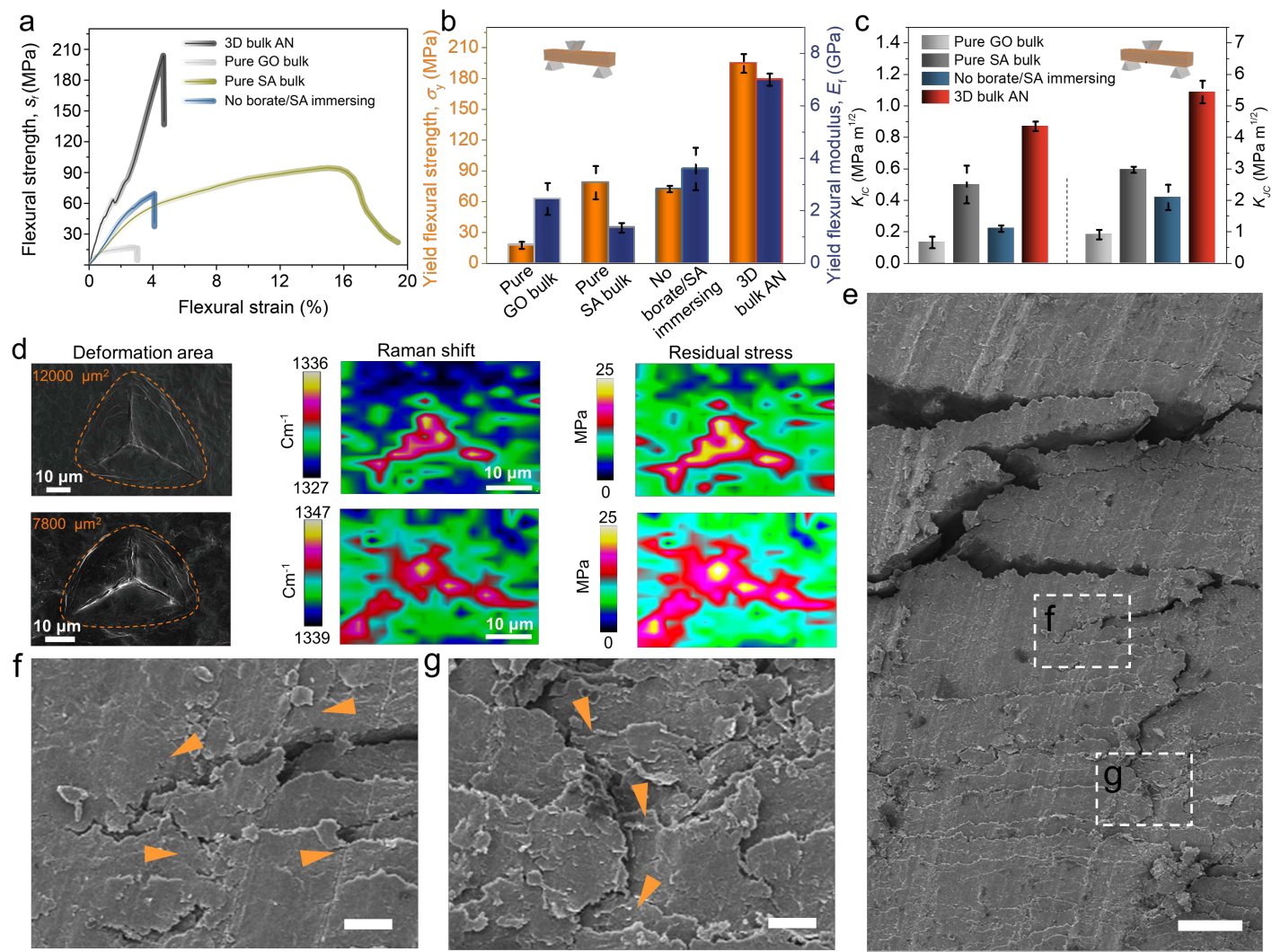

Fig. 4 | Mechanical properties, sharp contact indentation, residual stress fields, and microcrack deflection/crack bridging of the as-fabricated GML bulk materials. a, Flexural stress-strain curves, corresponding to the loading in the plane direction (see Supplementary Fig. 23). b, Flexural strength and stiffness. c, Comparison of fracture toughness for crack initiation $\left(K_{\mathrm{Ic}}\right)$ and stable crack propagation $\left(K_{\mathrm{Ic}}\right)$, respectively. d, Comparison for post-indentation SEM images of pure GO and GML bulk, corresponding to Raman mapping for visualization of residual stress fields around indents of the two typical samples, respectively. Relative intensity of the D band of GO (left); peak shift of the D band (middle); and residual stress fields as computed from the relative peak intensity and peak shift (right). e, Long-range crack propagation path, showing a serrated line and crack deflection. Scale bar: $50 \mu \mathrm{m}$. f,g At a higher magnification, crack deflection, branching, and multiple cracking around the propagating secondary crack tip (f) and toward the end of a main crack tip (g). Orange arrows indicate crack trajectories. Scale bars, $10 \mu \mathrm{m}$. 
Opening mode

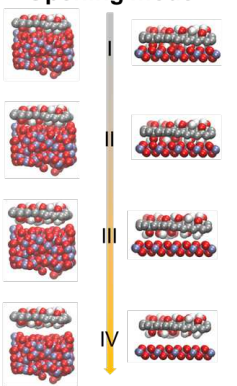

C Nanoscale structure

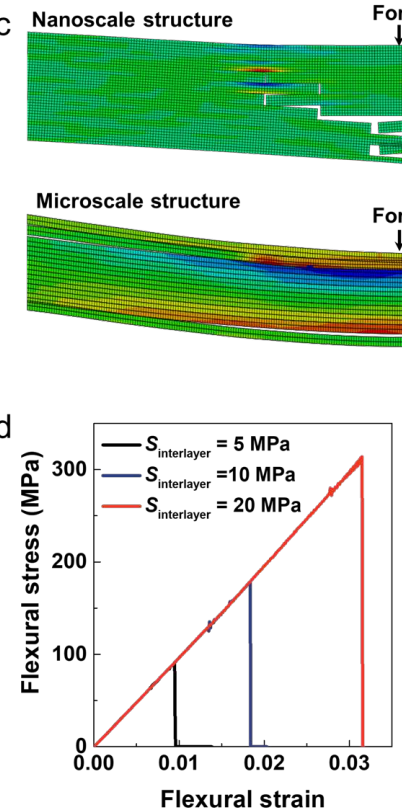

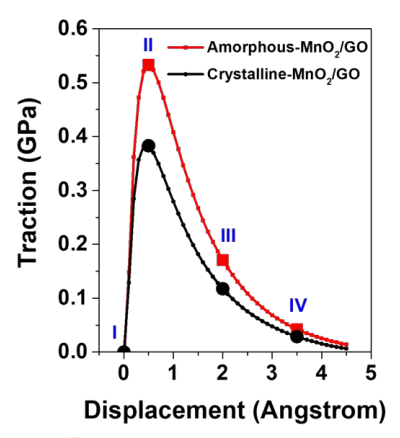

Force
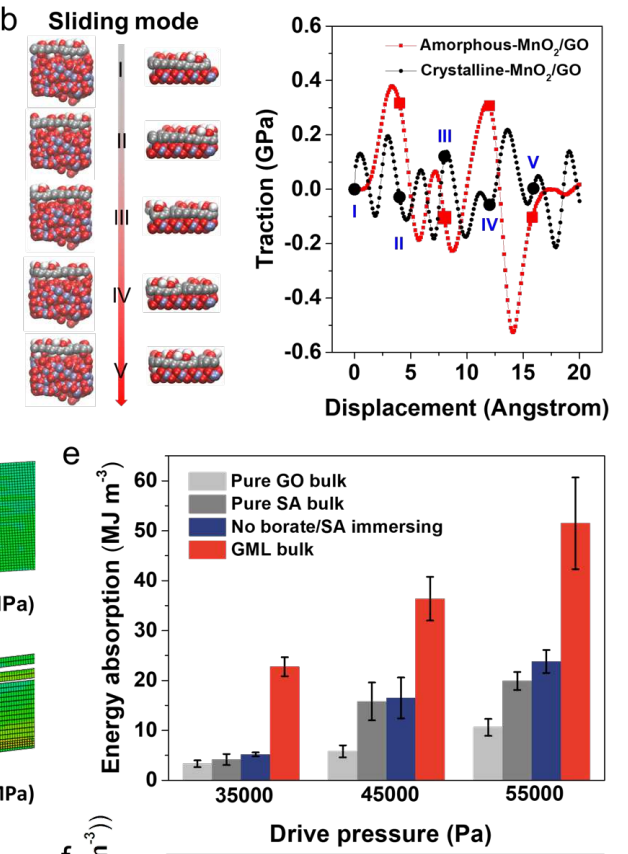

$-41.6-28.6-15.6-2.61+10.4+16.9+36.4$

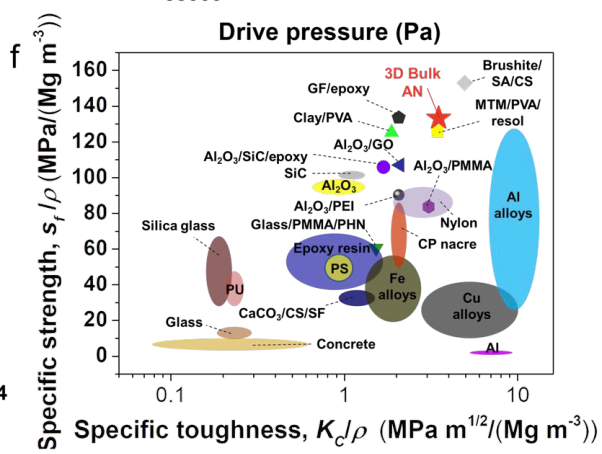

Fig. 5 | Multiple reinforcing mechanisms acting at multiple length scale by molecular dynamics (MD) and finite element (FE) simulations, impact resistance properties, and comparison of mechanical performance. a, MD simulations for snapshots (left) of the opening-mode separation processes of amorphous- $\mathrm{MnO}_{2} / \mathrm{GO}$ interface and crystalline- $\mathrm{MnO}_{2} / \mathrm{GO}$ interface system, respectively, which corresponding to the scattered points in the plot of traction-displacement behavior (right). The separation distances between $\mathrm{MnO}_{2}$ and $\mathrm{GO}$ are $0,0.5,2$, and $3.5 \AA$ in snapshots I, II, III, and IV. b, MD simulations for snapshots (left) of the sliding-mode separation processes of amorphous- $\mathrm{MnO}_{2} / \mathrm{GO}$ interface and crystalline- $\mathrm{MnO}_{2} / \mathrm{GO}$ interface system, respectively, which corresponding to the scattered points in the plot of traction-displacement behavior (right). The sliding distances between $\mathrm{MnO}_{2}$ and $\mathrm{GO}$ are $0,4,8,12$, and $16 \AA$ in snapshots I, II, III, IV, and V. c, FE simulations for stress nephograms of the fracture modes of the nanoscale hetero-phase reinforcing structure (upper) and the microscale lamellar structure (lower), respectively. d, FE simulations for variations of the effective flexural stress-strain curves of the microscale lamellar model dependent on different parameters of interface strength (left, 5-20 MPa) and lamella strength (right, 100-400 MPa), respectively. e, Impact resistance for the asprepared bulk materials at different initial drive pressures, including pure GO, pure SA, no borate/SA immersing, and GML bulk, derived from SHPB experiments. f, Ashby plot of specific toughness versus specific strength for many engineering and natural 
materials ${ }^{40}$. The GML bulk has specific strength or toughness similar to those of graphene foam/epoxy (GF/epoxy), clay/polyvinyl alcohol (clay/PVA), and $\mathrm{Al}_{2} \mathrm{O}_{3} / \mathrm{SiC} /$ epoxy. For the detailed data, see Supplementary Table 6 in SI. 


\section{Supplementary Files}

This is a list of supplementary files associated with this preprint. Click to download.

- 2Dto3DSI20210812.docx

- SupplementaryMovieS1.mp4

- SupplementaryMovieS2.mp4

- SupplementaryMovieS3.mp4 